\title{
Mining the Relationship between Geotagged Images and Facilities
}

\author{
Ching-Yu Huang, Henry Choque, and Brany Suero Benitez
}

\begin{abstract}
In recent years, many of the latest smartphones or mobile devices currently in the market come with built-in cameras and GPS systems. These devices can geotag and store critical geo-information along with the photos. This crucial metadata information will be used in the development for an algorithm that will identify the relationship between geotagged images and facilities (buildings). The development of this technology generates massive amounts of geo-data which could be very helpful to public and authorities on large campuses or cities. This research proposes methods to mine and construct the relationship between polygon points of buildings and the geo-information on huge sets of images, and then determine whether these buildings could be shown on the pictures or not. In order to test the methods, many geotagged images will be taken throughout the Kean University campus.
\end{abstract}

Index Terms - Geotagging, latitude, longitude, orientation, augmented reality.

\section{INTRODUCTION}

In the last few years, photos taken with smartphones have outnumbered photos taken with traditional cameras as more and more people use the built-in cameras in their phones. These devices come with special sensors that allow for additional data such as geographic information via GPS to be added to the metadata of these photos. Once a picture is taken, the camera registers the location the photo was taken in the form of latitude, longitude, altitude, direction as well as device names, taken date and time, resolution, ISO, exposure, shutter speed and other EXIF information. Recently, there has been a dramatic increase in the number of people using the mobile devices to take pictures and share with other people. The geo-location information for tagging pictures can be kept or removed when the images are transformed such as resizing, or uploaded to social media websites such as Flickr or Facebook.

The location that is automatically attached to a photo by a GPS enabled device reflects the position from where the photo was taken. Modern cameras or smartphones are equipped with an internal compass, which enriches the information recorded by including the orientation towards true north or magnetic north. A geo-semantic modeling was proposed to provide a powerful capability to learn visually from historical geo-tagged photos [1]. Geotagging photos can also be used in Computer Vision and multimedia, and virtual reality [2], [3].

Manuscript received September 5, 2015; revised March 15, 2016.

Ching-Yu Huang is with the Department of Computer Science, Kean University, USA (e-mail: hcy100@gmail.com).

Henry Choque is with Costume Supercenter in Edison, New Jersey, USA.

Brany Suero Benitez is with New Jersey Institute of Technology, USA.
A framework was proposed to estimate the camera viewing direction of a single geotagged photo in any environment [4]. For searching and tracking suspects, authorities can ask people to upload their geotagged photos. In order to let people know the city or campus better, people upload and share their pictures online. However, both cases will require methods that can automatically process a huge amount of geotagged images and identify the relationship between images and facilities. In this paper, we make use of the geo-information in order to develop an algorithm for identifying the relationship between geotagged images and facilities (buildings) located inside the Kean University campus. Overall the accuracy of these coordinates is approximately 5 to 10 meters [5] and meets our data requirements.

\section{Rule BASED METHODS}

For this research, we are using hundreds of geotagged images which have been uploaded to a database constructed using MySQL for easy accessing. The database runs under the Apache web server. For purposes of this research, we are also assuming that all photos have a 45 -degree angle view and are within the 5 to 10 -meter accuracy previously mentioned. For the campus buildings, GPS coordinates were obtained through Google Map [6]. PHP is the computer language used for this project because there free mathematical functions to retrieve geotagged information from photos and calculate the geometric data [7].

We proposed four specific algorithms and rules to identify the relationship between geotagged images and facilities. The first rule excludes any photos that were too far away from the target location. The second rule excludes any photos that were not facing the target location. The third rule excludes any photos that were blocked by another building. The fourth and final rule determine whether or not a photo had an overlap with another photo. Fig. 1 shows some photos, a partial campus map, and the 4 rules referring to a particular target location.

\section{A. Rule 1-Distance}

If a photo is too far from a target location, it might not contain the searching objects or the resolution might not be good enough to identify the objects. Since the uploaded photos could be from randomly located places, it is necessary to filter out unnecessary photos to narrow the amount of processing photos for next steps. To exclude a given photo that is $N \mathrm{ft}$ (500ft in our example) away from the Target Location (Willis Hall in our example), we implemented what is known as the 'haversine' formula [8]. From this formula, we define distance as computing the 
lengths of great circle arcs connecting pairs of points on the surface of a sphere (earth). We compute the azimuths of the second point in each pair with respect to the first (the angle where the arch crosses the meridian containing the first point). Since the input latitudes and longitudes can be scalars or arrays of equal size, they must be expressed in degrees.

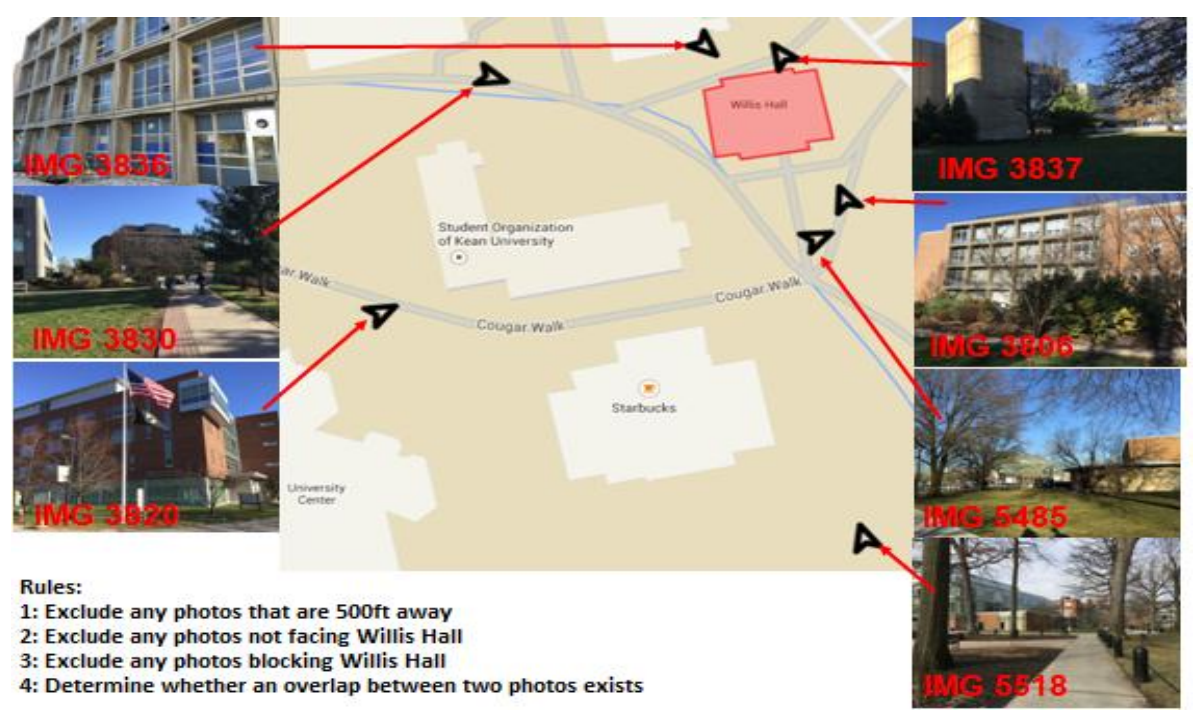

Fig. 1. The test photos on Kean campus.

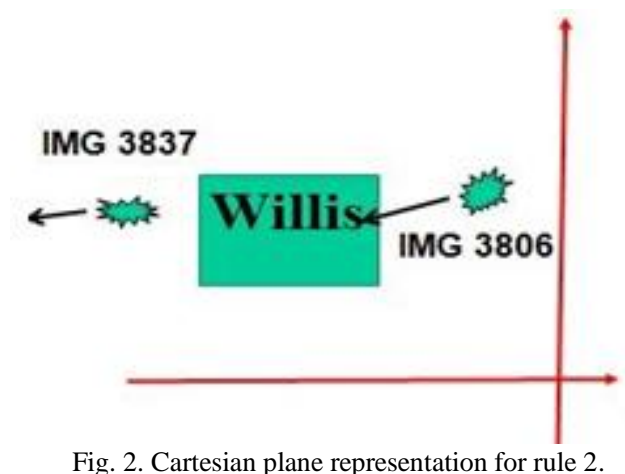

\section{B. Rule 2-Direction}

The photos will not contain the objects if they are not facing the target location, even if they are very close to the target location. So, the second rule was adopted to exclude any photos passing Rule 1 but not facing target location (Willis Hall in our example). First, a geotagged photo has a direction $b$. The bearing $b$ is defined to be the angle between two given points on earth's surface. Both $a$ and $b$ are assumed that degree 0 means facing north. If $|a-b|<c$, the photo is considered facing the building where $\mathrm{c}$ is a half of camera angle ( $c=25$ in our example). Two photos shown on Fig. 1 are examples. From the bearing between photos and buildings and direction of the photo, we can see the IMG3837 direction is not facing Willis Hall, and IMG3806 is facing Willis Hall (See Fig. 2).

\section{Rule 3-Blocking}

A photo might not contain the searching objects because other buildings could be blocking the objects even if the photo meets Rule 1 and 2 . The third rule needs to exclude any pictures that are blocked by other buildings. To accomplish this, the polygon points (GPS coordinates) of the building are required and they can manually be retrieved from Google maps. These points are passed to a polygon array to form the building. A new algorithm is applied to determine if the segment $(P n P m)$ of any two polygon points $P n$ and $P m$ intersect with the line $(P i P b)$ between the photo $P i$ and building center $P b$. As shown in Fig. 3, the line from photo IMG3820 to Willis Hall has two intersections (marked in a black dot). Therefore, photo IMG3820 won't contain Willis Hall.

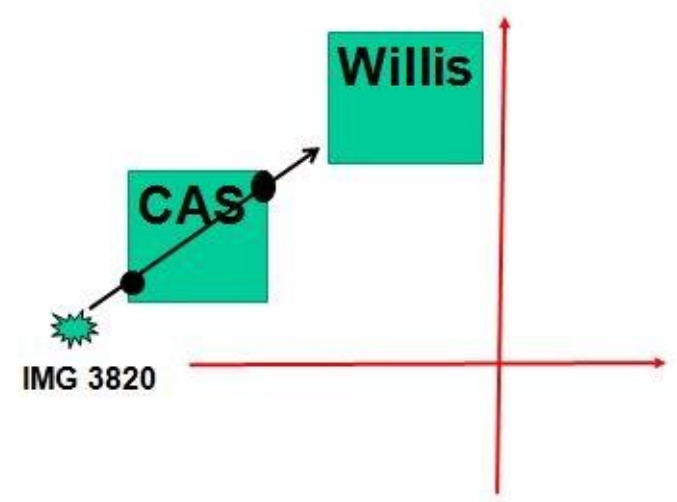

Fig. 3. Cartesian plane representation for rule 3.

\section{Rule 4-Overlapping}

When a photo meets Rule 1, 2 and 3, it should contain the target object. However, the photo only shows an angle of the object. In many cases, it is very important to have different views of the objects from different times or angles of other photos. This will require a new algorithm to identify if any photos could possibly overlap and have the same objects. The fourth algorithm is adopted to achieve this goal by checking if the angles $A i 1$ and $A i 1$ intersect each other where $A i 1, A i 2$ are the angles of photo $i 1$ and $i 2$ respectively with the assumption $i 1$ and $i 2$ meet rules 1,2 and 3 . The left image on Fig. 4 shows that photo IMG5485 is not facing the same direction as photo IMG3806. So, they are not overlapped and won't possibly have same objects. The photos IMG3806 and IMG5517 on the right image of Fig. 4 have same objects because the photos are overlapped. 


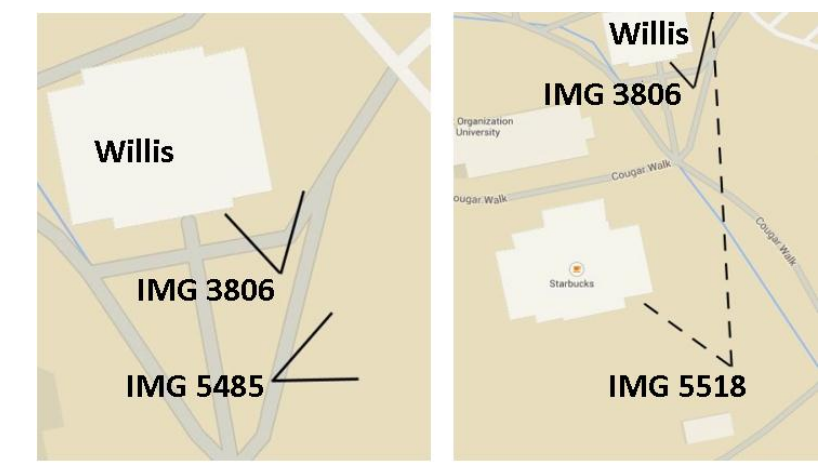

Fig. 4. Rule 4 no angle intersection; Rule 4 match angle intersection found.

\section{ANALYSIS OF EXPERIMENTAL RESULTS}

In our approach, we used the above rules to obtain our results. The algorithms were designed around these rules and were tested to prove their accuracy. For our first goal, we can observe that any pictures outside of the 500ft range have been excluded since these pictures are not very accurate. For our second rule, image IMG 3837 does not face our primary target which is Willis Hall and is consequently excluded as well. Image IMG 3820 direction does face Willis Hall but is being blocked by another building, in this the case the CAS (Center for Academic Success) building. For our final rule, we try to determine whether two photos intersect by finding the point of intersection between these two images. In our case, we can see that IMG 5518 and IMG 3806 face the direction and despite the fact that one photo is much farther away than the other, an intersection does occur. Several hundreds of photos were available on the database, but a set of 20 photos was picked to test rules 1,2 and 3 . The results were manually verified and shown on Table I.

For Rule 1, we entered two coordinate points and used the earlier described method to calculate the distance between these two points. The results were manually verified by comparing the results with similar algorithms found online such as Google maps distance feature. For Rule 2, similar algorithms were not found to compare our results, but we were able to visually verify our results by comparing our given method results from the orientation retrieved from the metadata stored in the picture. In the above picture, for example, by observing the arrows we can determine whether it faces the target building and compare this with our algorithm.

For Rule 3, we discarded any pictures that although were facing our main target, were also being blocked by another building. For this method, the polygon points of the blocking facility were retrieved from Google Maps in order to create an outline image of the building. If the given angle direction of the photo intersected any of these points, then we could safely assume that a blocked had occurred. For Rule 4, we had to determine whether an intersection between two given photos had occurred. In order to do this, we had to find the point of intersection between these two photos. Once a point of intersection was found, it meant that an intersection had occurred. There was a flaw in the algorithm, however, basically due to the fact that certain intersections could potentially occur behind or even within a facility. In this case, the two photos are not considered overlapped. Table II shows the result of the 190 comparisons among 20 photos.

TABLE I: RESULTS OF RULE 1, 2 AND 3

\begin{tabular}{|c|c|c|c|}
\hline Results & Total \# of photos & Total \# of errors & Accuracy \\
\hline Rule 1 & 20 & 0 & $100 \%$ \\
\hline Rule 2 & 20 & 2 & $90 \%$ \\
\hline Rule 3 & 18 & 3 & $83.3 \%$ \\
\hline
\end{tabular}

\begin{tabular}{|c|c|c|c|}
\hline \multicolumn{5}{|c|}{ TABLE II: RESULTS OF RULE 4} \\
\hline $\begin{array}{c}\text { Total \# of } \\
\text { comparisons }\end{array}$ & $\begin{array}{c}\text { Total \# of } \\
\text { overlaps }\end{array}$ & $\begin{array}{l}\text { Total \# of } \\
\text { overlap errors }\end{array}$ & Accuracy pct \\
\hline 190 & 86 & $5 / 24$ & $80 \%$ \\
\hline
\end{tabular}

\section{CONCLUSION}

How to identify the relationship between photos and buildings are very important in mining spatial data and constructing an Augmented Reality (AR) Information system. Authorities can use the relationship to track suspects through a large volume of photos uploaded by the public. People can use AR App on their mobile device to face a facility or an open space to see the event's information, including the multimedia supplements, without typing in any URL. The 3-dimension geographic information and the direction the user is facing can be constructed by the photo relationship to identify the target facility to retrieve the corresponding events and activities.

This research showed how geotagged information can be used in a variety of ways, and they are very helpful for new students, faculty, staff and visitors to become familiar with the campus as well as for everyone to find out about events such as open houses, seminars, exhibits, club activities, and competitions.

All the obtained results were thoroughly checked in an effort to obtain the highest possible accuracy. This research is in its beginning stage and sets the foundation for future students who want to continue researching this type of technology. Our research will eventually help construct a campus view for augmented reality or even help authorities automatically narrow down/identify suspects by collecting camera photos from the general public.

However, the geotagged information can be also used by predators to track and invade people, especially the minors or innocent students. Many people like to use their cellular phone to take and share pictures on social media. In order to protect people's privacy and safety, we strongly suggest that all websites should give users options to remove the geotagged information when they upload and share personal photos. This option could be also implemented in browser settings to automatically remove the geo-tagged information when the browsers upload the photos to any websites so users will not need to rely on if websites provide the options.

\section{REFERENCES}

[1] J. R. Smith, "What happens where?" in Proc. the 2nd ACM International Workshop on Geotagging and Its Applications in Multimedia, Barcelona, Catalunya, Spain, October 21-25, 2013.

[2] J. B. Luo, D. Joshi, J. Yu, and A. Gallagher, Geotagging in Multimedia and Computer Vision - A Survey, 2011, pp. 1-19.

[3] C. Hang, A. Gallagher, and T. Chen, "GPS refinement and camera orientation estimation from a single image and a 2D map," in Proc. 2014 IEEE Conference on Computer Vision and Pattern Recognition Workshops, 2014. 
[4] M. Park, J. Luo, R. T. Collins, Y. Liu, "Beyond GPS: Determining the camera viewing direction of a geotagged image," in Proc. the International Conference on Multimedia, Firenze, Italy, October 2529, 2010.

[5] B. Christine. On the (In-) Accuracy of GPS Measure of Smartphones: A Study of Running Tracking Applications, Dec. 2013.

[6] Google Street View Image API, Google Developers, p. 21, May 2015.

[7] Trying to Calculate Distance between Locations Using PHP While and for Loops, Aug. 2, 2013.

[8] C. Veness. Movable type scripts. Calculate Distance and Bearing between Two Latitude/Longitude Points Using Haversine Formula in JavaScript. (March 22, 2015). [Online]. Available: http://www.movable-type.co.uk/scripts/latlong.html

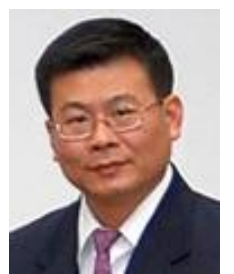

Ching-Yu Huang is an assistant professor of the Department of Computer Science at Kean University since September 2014. Dr. Huang was born in Taiwan and received a Ph.D. degree in computer and information science from New Jersey Institute of Technology, Newark, New Jersey, USA in January 1998.

Prior to joining Kean University, Dr. Huang had more than 16 years of experience in the industry and academics in software development and R\&D in bioinformatics. His researches are focus on SNP genotype calling and cluster detection, image processing and pattern recognition, especially in microarray and fingerprint; geotagged images and location information reconstruction, database application development, data processing automation, e-learning, educational multimedia, methodology, and online tools for secondary schools and colleges. Dr. Huang has more than 20 publications in journals and conferences and more than 20 presentations in workshops and invited lectures.

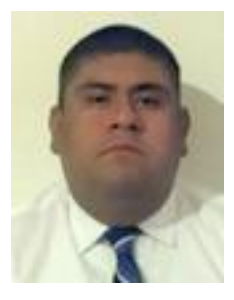

Henry Choque was born in Lima, Peru. He received his B.S. degree in computer science from Kean University, Union, New Jersey in 2015. While in college, he completed a summer internship at MTF (Musculoskeletal Transplant Foundation) as an IT administrator where he gained some valuable experience in system troubleshooting, computer networking and system imaging. After graduation, he joined Costume Supercenter in Edison, New Jersey as a Jr. IT System Administrator.

$\mathrm{He}$ is a member of the LSAMP program and continues to pursue his interest in computer programming, with emphasis on PHP, Java and database development.

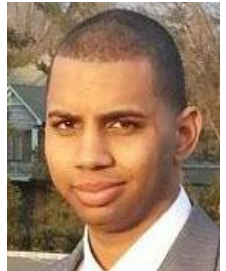

2009.
Brany Suero graduated in May 2015 with a dual major of a B.S degree in computer Science and B.A. degree in mathematics from Kean University, Union, NJ. Currently, he is pursuing his master degree in computer science at New Jersey Institute of Technology. Brany is passionate for big data, software development (involving mathematical procedures) and new technology. Brany Suero came to the US in 2008 and joined Kean University in 